\title{
PRODUÇÃO DE SULFATO DE AMÔNIO DUPLAMENTE MARCADO COM OS ISÓTOPOS ESTÁVEIS ${ }^{15} \mathrm{~N}$ E ${ }^{34} \mathrm{~S}$
}

\author{
Everaldo Maximo, José Albertino Bendassolli*, Paulo César Ocheuze Trivelin, Alexssandra Luiza Rodrigues Molina \\ Rossete, Claudinéia Raquel de Oliveira e Clelber Vieira Prestes \\ Centro de Energia Nuclear na Agricultura, Universidade de São Paulo, CP 96, 13400-970 Piracicaba - SP
}

Recebido em 3/2/04; aceito em 27/8/04; publicado na web em 4/2/05

\begin{abstract}
PRODUCTION OF AMMONIUM SULFATE DOUBLY LABELED WITH THE ${ }^{15} \mathrm{~N}$ AND ${ }^{34} \mathrm{~S}$ STABLE ISOTOPES. The purpose of this work was the production of ammonium sulfate double labeled with ${ }^{15} \mathrm{~N}$ and $\left.{ }^{34} \mathrm{~S}\left(\left({ }^{15} \mathrm{NH}_{4}\right){ }_{2}^{34} \mathrm{SO}_{4}\right)\right)$, employing the ion exchange technique in two different processes. The first one was carried out using $\mathrm{Na}_{2}{ }^{34} \mathrm{SO}_{4}$ and $\left({ }^{15} \mathrm{NH}_{4}\right)_{2} \mathrm{SO}_{4}$ previously enriched. It was possible to obtain about $54 \mathrm{~g}$ of $\left({ }^{15} \mathrm{NH}_{4}\right)_{2}{ }^{34} \mathrm{SO}_{4}$ from $70.0 \mathrm{~g}$ of $\mathrm{Na}_{2}{ }_{2}^{34} \mathrm{SO}_{4}$ and $64.2 \mathrm{~g}$ of $\left({ }^{15} \mathrm{NH}_{4}\right)_{2} \mathrm{SO}_{4}$. The second method involved the production of $\mathrm{H}_{2}^{34} \mathrm{SO}_{4}$, by ion exchange, and its subsequent reaction with ${ }^{15} \mathrm{NH}_{3 \text { (aq) }}$, using a distillation system, to yield $58 \mathrm{~g}$ of $\left({ }^{15} \mathrm{NH}_{4}\right)_{2}{ }^{34} \mathrm{SO}_{4}$ from $43.1 \mathrm{~g}$ of $\mathrm{H}_{2}{ }^{34} \mathrm{SO}_{4}$.
\end{abstract}

Keywords: nitrogen; sulfur; ion exchange.

\section{INTRODUÇÃO}

O nitrogênio e o enxofre são dois nutrientes essenciais aos vegetais e animais, por fazerem parte de aminoácidos e proteínas relacionados às mais variadas funções bioquímicas ${ }^{1}$.

$\mathrm{Na}$ área agronômica, sobretudo na nutrição de plantas, avaliações da dinâmica dos elementos $\mathrm{N}$ e $\mathrm{S}$ contribuem para importantes avanços no aumento da produtividade e qualidade dos produtos agrícolas. O S é importante quando se considera a qualidade dos produtos de origem agrícola ${ }^{2}$. Estudos indicaram que o sulfato de amônio, como fornecedor de $\mathrm{N}$ e $\mathrm{S}$, está claramente contribuindo para o aumento da produtividade ${ }^{3-6}$. O aumento da eficiência de utilização do $\mathrm{N}$ aplicado às culturas agrícolas, entre outros fatores, é atribuído à existência de sinergismo com o $\mathrm{S}^{7}$. A utilização do sulfato de amônio como fertilizante tem sido preconizada em relação à uréia, em aplicações na superfície e em solo não alcalino, primeiro por não apresentar perdas expressivas de amônia por volatilização e segundo, por fornecer o nutriente $S$, uma vez que a uréia não contém o nutriente mesmo apresentando menor custo por unidade de $\mathrm{N}^{3,5,8-10}$. A uréia quando aplicada em superfície, sobre a palhada que recobre o solo, pode diminuir muito sua eficiência agronômica, pelas perdas de amônia por volatilização ${ }^{11-14}$. A importância da aplicação do enxofre em culturas agrícolas tem sido evidenciada nestes últimos anos pela redução de sua disponibilidade aos vegetais (deficiência de $\mathrm{S}$ em muitas regiões), verificada principalmente pelo uso de fertilizantes concentrados em NPK ${ }^{4,15-18}$.

Naturalmente ocorrem quatro isótopos estáveis de enxofre $\left({ }^{32} \mathrm{~S}\right.$, $\left.{ }^{33} \mathrm{~S},{ }^{34} \mathrm{Se}^{36} \mathrm{~S}\right)$ e dois isótopos de nitrogênio $\left({ }^{14} \mathrm{~N} \mathrm{e}{ }^{15} \mathrm{~N}\right)$. Estes isótopos apresentam abundância isotópica natural de 95,$02 ; 0,75 ; 4,21$ e $0,02 \%$ em átomos, respectivamente, para os isótopos ${ }^{32} \mathrm{~S},{ }^{33} \mathrm{~S},{ }^{34} \mathrm{~S}$ e ${ }^{36} \mathrm{~S}$. Para o nitrogênio, a abundância natural é dada por 99,73 e 0,37\% em átomos para ${ }^{14} \mathrm{Ne}^{15} \mathrm{~N}$, respectivamente ${ }^{19}$. A existência dos isótopos raros mais pesados, do enxofre ${ }^{34} \mathrm{~S}$ e do nitrogênio ${ }^{15} \mathrm{~N}$, possibilita a produção de compostos marcados ou enriquecidos nestes isótopos, caracterizados por apresentarem abundâncias isotópicas acima da natural. Estes compostos por sua vez possibilitam o uso da técnica isotópica de traçador, na elucidação de aspectos relacionados à utili-

*e-mail: jab@cena.usp.br zação destes nutrientes pelas plantas cultivadas, sendo também possível a realização de estudos de metabolismo bioquímico em humanos, a partir de moléculas apirogênicas.

Isótopos radioativos de enxofre e nitrogênio constituem alternativas em estudos do ciclo destes elementos. Pesquisas relacionadas com nutrição de $\mathrm{S}$ em plantas são geralmente facilitadas com o uso do radioisótopo ${ }^{35} \mathrm{~S}^{20-24}$. No entanto, apesar de os radioisótopos apresentarem vantagens relacionadas ao menor custo e elevada sensibilidade de detecção, o uso de isótopos estáveis tem sido preconizado sempre quando possível, sobretudo em experimentação de campo e com seres vivos. O radioisótopo do nitrogênio que apresenta meia vida mais longa é o ${ }^{13} \mathrm{~N}$, com apenas $9,7 \mathrm{~min}$, inviabilizando seu emprego na maioria dos estudos.

O radioisótopo ${ }^{35} \mathrm{~S}$ é um emissor $\beta-(0,167 \mathrm{Mev})$, com meia vida de 87,2 dias $^{19}$ e tem sido empregado como traçador em estudos de ciclagem de enxofre no sistema solo-planta. Pesquisas de nutrição de planta envolvendo o nutriente $\mathrm{S}$ têm sido realizadas empregando o radioisótopo ${ }^{35} \mathrm{~S}$. A utilização do isótopo estável ${ }^{34} \mathrm{~S}$, em estudos do ciclo do enxofre, requer a disponibilidade de compostos enriquecidos no isótopo e de espectrômetro de massas para a determinação isotópica. A utilização de isótopos estáveis demanda custos superiores, quando comparada com experimentos em que se utilizam compostos radiomarcados com ${ }^{35} \mathrm{~S}$ empregando a técnica de cintilação líquida para detecção. O composto $\left({ }^{15} \mathrm{NH}_{4}\right){ }_{2}{ }^{34} \mathrm{SO}_{4}$ enriquecido a $10 \%$ nos isótopos ${ }^{15} \mathrm{~N} \mathrm{e}^{34} \mathrm{~S}$ tem preço FOB da ordem de US\$ 33.00 (trinta e três dólares) por grama no mercado internacional. No entanto, apesar de o fator custo representar uma desvantagem, esta é superada pelos aspectos de segurança, ausência de resíduos radioativos (cintilação) e, principalmente, confiabilidade nos resultados em experimentos de longo prazo (acima de 20 meses). Cabe ressaltar que há uma tendência internacional voltada para o uso dos isótopos estáveis em trabalhos de campo, sempre que possível, restringindo desta forma o uso do ${ }^{35} \mathrm{~S}$ em experimentos dessa natureza ${ }^{2,15,25}$. Com relação aos isótopos estáveis, o uso de ${ }^{34} \mathrm{~S}$ como traçador é comprometido devido ao custo e à dificuldade em se obter os compostos marca$\operatorname{dos}^{28}$. Na literatura foi mencionado que, na década de 60, os EUA e a Rússia estavam desenvolvendo método de enriquecimento de ${ }^{34} \mathrm{~S}$ e que os laboratórios poderiam fornecer compostos a preços acessíveis, expectativa esta que não se concretizou ${ }^{26}$. Foi destacada também, a importância da alternativa de utilização do isótopo estável do 
enxofre (principalmente ${ }^{34} \mathrm{~S}$ ) em pesquisas envolvendo o nutriente, em substituição aos compostos radiomarcados com ${ }^{35} \mathrm{~S}$. Em trabalho envolvendo ${ }^{34} \mathrm{~S}$ em estudos de absorção e redistribuição de enxofre na cultura de trigo ${ }^{2}$, pôde-se destacar a importância da técnica com uso do traçador estável ${ }^{34} \mathrm{~S}$. Entretanto, os autores mencionam que a metodologia analítica para determinação da abundância isotópica de ${ }^{34} \mathrm{~S}$ em compostos com alto enriquecimento no isótopo não foi devidamente testada. Destacou-se que os trabalhos com utilização do traçador ${ }^{34} \mathrm{~S}$ são raros, e um primeiro trabalho somente foi produzido há uma década atrás ${ }^{27}$.

No Brasil, o primeiro estudo de nutrição de plantas utilizando a técnica isotópica com ${ }^{34} \mathrm{~S}$ foi realizado no Centro de Energia Nuclear na Agricultura ${ }^{15}$. Os autores avaliaram a utilização do enxofre adicionado ao solo, como fertilizante, para arroz (Oryza sativa L.) e para crotalária (Crotalaria juncea L.).

Pelo exposto, o desenvolvimento metodológico para produção de compostos marcados em seus isótopos estáveis tem sido estimulado, por possibilitar a obtenção de informações refinadas sobre o ciclo de seus elementos constituintes. Neste sentido, a síntese de fertilizantes marcados nos nutrientes $\mathrm{S}$ e $\mathrm{N}$ é de potencial interesse em pesquisas agronômicas. $\mathrm{O}$ sulfato de amônio duplamente marcado nos isótopos ${ }^{34} \mathrm{Se} \mathrm{e}^{15} \mathrm{~N}\left(\left({ }^{15} \mathrm{NH}_{4}\right)_{2}{ }^{34} \mathrm{SO}_{4}\right)$, cuja obtenção foi o objetivo do trabalho, constitui-se numa importante alternativa para avaliações simultâneas da dinâmica do enxofre e do nitrogênio no sistema solo-planta, possibilitando estudos comparativos considerando as duas variáveis e sua inter-relação, através de isotopia multivariada. A produção do composto duplamente marcado foi encorajada pelo seu potencial de utilização na compreensão do ciclo do N e S simultaneamente. Outro fator importante está relacionado ao domínio de tecnologia de separação e produção de compostos enriquecidos nos isótopos ${ }^{34} \mathrm{~S}$ e ${ }^{15} \mathrm{~N}$, que notadamente restringe-se aos países desenvolvidos.

\section{PARTE EXPERIMENTAL}

\section{Reagentes, isótopos e soluções}

Todas as soluções foram preparadas com reagentes de qualidade química analítica e com água desionizada.

$\mathrm{O}\left({ }^{15} \mathrm{NH}_{4}\right)_{2} \mathrm{SO}_{4}$ com enriquecimento na faixa de $1 \mathrm{a} 80 \%$ em átomos de ${ }^{15} \mathrm{Ne}$ o $\mathrm{Na}_{2}{ }^{34} \mathrm{SO}_{4}$ enriquecido com 5 a $17 \%$ em átomos de ${ }^{34} \mathrm{~S}$, produzidos no próprio Laboratório de Isótopos Estáveis do CENA/ $\mathrm{USP}^{25,28,30}$, foram utilizados como fonte dos isótopos pesados ${ }^{34} \mathrm{~S}$ e ${ }^{15} \mathrm{~N}$, para a produção do sulfato de amônio duplamente marcado $\left(\left({ }^{15} \mathrm{NH}_{4}\right)_{2}{ }^{34} \mathrm{SO}_{4}\right)$. Com o objetivo de verificar possível fracionamento isotópico, no processo de troca iônica, utilizou-se de $\mathrm{Na}_{2}{ }^{34} \mathrm{SO}_{4}$ enriquecido com $8,4 \%$ em átomos de ${ }^{34} \mathrm{~S}$ e $\left({ }^{15} \mathrm{NH}_{4}\right)_{2} \mathrm{SO}_{4} \mathrm{com} 15,2 \% \mathrm{em}$ átomos de ${ }^{15} \mathrm{~N}$.

\section{Linha de produção}

A linha de obtenção do $\left({ }^{15} \mathrm{NH}_{4}\right)_{2}^{34} \mathrm{SO}_{4}$ foi composta de uma coluna acrílica com $2,1 \mathrm{~cm}$ de diâmetro interno e $150 \mathrm{~cm}$ de altura, preenchida até $20 \mathrm{~cm}$ do topo com resina catiônica fortemente ácida, tipo poliestireno-divinilbenzeno Dowex 50W-X8 (grupo funcional sulfônico, malha 100-200 com diâmetro das pérolas de 74-149 $\mu \mathrm{m}$, capacidade de troca de $1,7 \mathrm{mmol} \mathrm{H}^{+} \mathrm{mL}^{-1}$ ). O volume de resina na forma $\mathrm{H}^{+}$equilibrada em água, totalizou $450 \mathrm{~mL}$. A Figura 1 representa um esquema da coluna utilizada, bem como as equações envolvidas no processo.

O mesmo sistema de coluna foi utilizado para a obtenção de

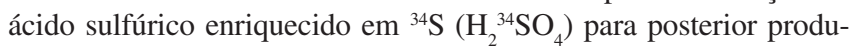
ção de $\left({ }^{15} \mathrm{NH}_{4}\right)_{2}{ }^{34} \mathrm{SO}_{4}$, a partir da reação do $\mathrm{H}_{2}^{34} \mathrm{SO}_{4}$ com ${ }^{15} \mathrm{NH}_{3(\text { aq })}$.

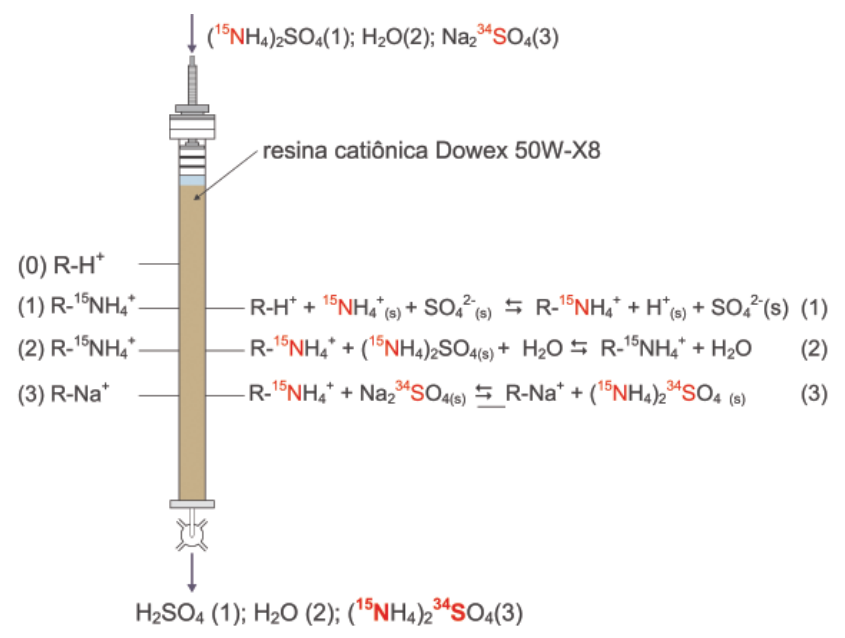

Figura 1. Coluna cromatográfica utilizada para produção de $\left({ }^{15} \mathrm{NH}_{4}\right)_{2}^{34} \mathrm{SO}_{4}$

\section{Procedimentos experimentais}

Antes do empacotamento na coluna, a resina foi lavada várias vezes com água desionizada, com a finalidade de retirar as impurezas e traços de resíduos orgânicos provenientes do processo de síntese. Posteriormente a resina foi acondicionada na coluna de acrílico e submetida ao choque eletrolítico, objetivando a ativação dos seus sítios ativos que foram saturados às formas $\mathrm{NH}_{4}^{+}\left(\mathrm{R}_{-} \mathrm{NH}_{4}^{+}\right)$e $\mathrm{H}^{+}(\mathrm{R}-$ $\mathrm{H}^{+}$), alternadamente, por 3 ciclos com solução $1,0 \mathrm{~mol} \mathrm{~L}^{-1}$ de $\left(\mathrm{NH}_{4}\right)_{2} \mathrm{SO}_{4}$ e $1,0 \mathrm{~mol} \mathrm{~L}^{-1}$ de $\mathrm{H}_{2} \mathrm{SO}_{4}$, respectivamente. Finalmente foi realizada a lavagem da resina, com a finalidade de eliminar o ácido sulfúrico contido em seu volume intersticial. O fluxo de soluções deu-se por gravidade, com os reservatórios localizados à $5 \mathrm{~m}$ de altura em relação à base da coluna.

\section{Produção de $\left({ }^{15} \mathrm{NH}_{4}\right)_{2}{ }^{34} \mathrm{SO}_{4}$ por troca iônica}

No topo da coluna de resina catiônica, inicialmente na forma R$\mathrm{H}^{+}$, foi admitido solução $0,5 \mathrm{~mol} \mathrm{~L}^{-1}$ de $\left({ }^{15} \mathrm{NH}_{4}\right)_{2} \mathrm{SO}_{4}$ visando a saturação dos sítios ativos da resina à forma $\mathrm{R}^{-15} \mathrm{NH}_{4}^{+}$, Eq. (1) da Figura 1. Após a carga com íons ${ }^{15} \mathrm{NH}_{4}^{+}$procedeu-se à lavagem da resina pela passagem de $\mathrm{H}_{2} \mathrm{O}$ desionizada, para eliminação da solução de $\left({ }^{15} \mathrm{NH}_{4}\right)_{2} \mathrm{SO}_{4}$ contida no volume intersticial, ficando a resina na forma R- ${ }^{15} \mathrm{NH}_{4}^{+}$equilibrada em água, conforme Eq. (2), Figura 1. Nesta etapa, o volume do efluente foi coletado em alíquotas de $100 \mathrm{~mL}$ para determinação da concentração de ${ }^{15} \mathrm{NH}_{4}{ }^{+}$em sistema de destilação por arraste a vapor.

Em sequiência, utilizando-se do método de cromatografia por deslocamento frontal, foi admitida uma solução eluente de $\mathrm{Na}_{2}{ }^{34} \mathrm{SO}_{4}$ no topo da coluna de resina. Este procedimento teve a finalidade de eluir os íons ${ }^{15} \mathrm{NH}_{4}^{+}$, ligados aos sítios ativos da resina, pelos íons trocadores $\mathrm{Na}^{+}\left(\mathrm{Na}_{2}^{4}{ }^{34} \mathrm{SO}_{4}\right)$ ficando esta na forma $\mathrm{R}-\mathrm{Na}^{+}$. Com esse procedimento obteve-se no volume eluído, solução de $\left({ }^{15} \mathrm{NH}_{4}\right){ }_{2}^{34} \mathrm{SO}_{4}$, conforme a Eq. (3) da Figura 1, que foi quantificada. A concentração de $\mathrm{Na}^{+}$no eluído foi também determinada, por fotometria de chama, objetivando-se verificar o ponto de ruptura do elemento, dado pelo momento em que o íon $\mathrm{Na}^{+}$é detectado no eluído, juntamente com o $\mathrm{NH}_{4}{ }^{+33}$.

Após a completa eluição dos íons amônio, encerrou-se o processo com a retirada do excesso de $\mathrm{Na}_{2}{ }^{34} \mathrm{SO}_{4}$, contido no volume intersticial da resina, sendo o mesmo recuperado.

Foram avaliadas três concentrações diferentes para a solução eluente de $\mathrm{Na}_{2}{ }^{34} \mathrm{SO}_{4}\left(30,50\right.$ e $\left.70 \mathrm{~g} \mathrm{~L}^{-1}\right)$ e os testes foram realizados em triplicata. 


\section{Produção de $\left({ }^{15} \mathrm{NH}_{4}\right)_{2}{ }^{34} \mathrm{SO}_{4}$ a partir do $\mathrm{H}_{2}^{34} \mathrm{SO}_{4}$ e $\left({ }^{15} \mathrm{NH}_{4}\right)_{2} \mathrm{SO}_{4}$}

$\mathrm{O} \mathrm{H}_{2}{ }^{34} \mathrm{SO}_{4}$ foi obtido a partir do $\mathrm{Na}_{2}{ }^{34} \mathrm{SO}_{4}^{28,29}$ empregando-se o mesmo sistema de coluna de resina catiônica descrito anteriormente.

Inicialmente, os sítios ativos da resina foram saturados à forma R- $\mathrm{H}^{+}$pela admissão de solução $0,5 \mathrm{~mol} \mathrm{~L}{ }^{-1}$ de $\mathrm{H}_{2} \mathrm{SO}_{4}$ (abundância isotópica natural em ${ }^{34} \mathrm{~S}-4,22 \%$ em átomos de ${ }^{34} \mathrm{~S}$ ) no topo da coluna. A solução efluente da resina foi recolhida em alíquotas de 100 $\mathrm{mL}$, determinando-se a concentração de $\mathrm{H}^{+}$por titulometria, com o objetivo de se verificar o ponto final de carga. Depois de saturados os sítios ativos da resina $\left(\mathrm{R}-\mathrm{H}^{+}\right)$, eliminou-se o excesso de $\mathrm{H}_{2} \mathrm{SO}_{4}$ do volume intersticial da resina pela lavagem com $\mathrm{H}_{2} \mathrm{O}$ desionizada. Em seguida, foi realizado o descompactamento das camadas de resina com água em processo de retrolavagem. Na etapa seguinte, os íons $\mathrm{H}^{+}$foram eluídos com solução eluente de $\mathrm{Na}_{2}{ }^{34} \mathrm{SO}_{4}$, sendo avaliadas (em triplicatas) as concentrações de $30,50 \mathrm{e} 70 \mathrm{~g} \mathrm{~L}^{-1}$. A Figura 2 ilustra as etapas realizadas na produção de $\mathrm{H}_{2}{ }^{34} \mathrm{SO}_{4}$.

Nos testes de produção de $\mathrm{H}_{2}{ }^{34} \mathrm{SO}_{4}$, os íons $\mathrm{H}^{+}$foram deslocados da resina para a fase solução e na presença do ânion sulfato ocorreu a produção do ácido. A quantificação da massa de $\mathrm{H}_{2}^{34} \mathrm{SO}_{4}$ no volume eluído (a cada $100 \mathrm{~mL}$ ) foi realizada por titulometria (nas alíquotas dos volumes). Nas mesmas amostras determinou-se a concentração de $\mathrm{Na}^{+}\left(\mathrm{g} \mathrm{L}^{-1}\right.$ de $\left.\mathrm{Na}\right)$ para se verificar quando ocorria a mistura entre o $\mathrm{H}_{2}{ }^{34} \mathrm{SO}_{4}$ e o $\mathrm{Na}_{2}{ }^{34} \mathrm{SO}_{4}$ no volume eluído, indicando a curva de ruptura do sódio.

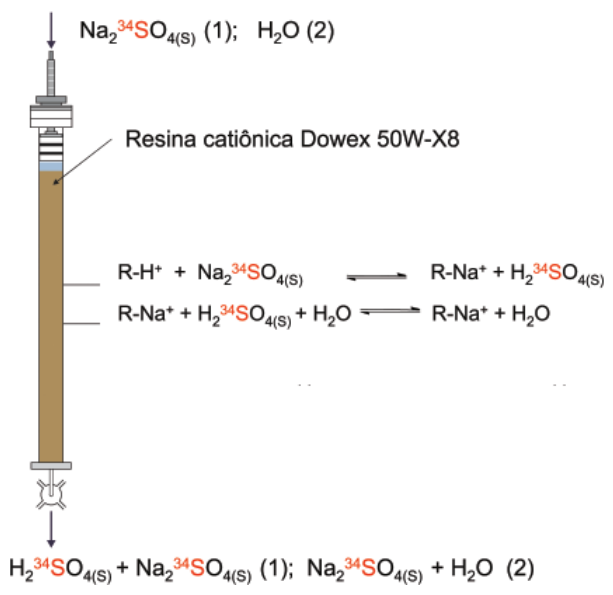

Figura 2. Sistema de produção de $\mathrm{H}_{2}^{34} \mathrm{SO}_{4}$ em coluna de resina catiônica

Partindo-se de soluções de $\mathrm{H}_{2}{ }^{34} \mathrm{SO}_{4}$ e $\left({ }^{15} \mathrm{NH}_{4}\right)_{2} \mathrm{SO}_{4}$ e utilizandose sistema de destilação (Figura 3), foi possível a obtenção do $\left({ }^{15} \mathrm{NH}_{4}\right){ }_{2}^{34} \mathrm{SO}_{4}$ de acordo com as reações (1) e (2).

$\left({ }^{15} \mathrm{NH}_{4}\right)_{2} \mathrm{SO}_{4}+2 \mathrm{NaOH} \leftrightarrows 2{ }^{15} \mathrm{NH}_{3 \mathrm{aq}}+\mathrm{Na}_{2} \mathrm{SO}_{4}$ $2{ }^{15} \mathrm{NH}_{3 \mathrm{aq}}+\mathrm{H}_{2}{ }^{34} \mathrm{SO}_{4} \leftrightarrows\left({ }^{15} \mathrm{NH}_{4}\right){ }_{2}^{34} \mathrm{SO}_{4}$

Inicialmente pesou-se o sal de $\left({ }^{15} \mathrm{NH}_{4}\right)_{2} \mathrm{SO}_{4}$ que foi dissolvido em água desionizada. A quantidade do sal $\left({ }^{15} \mathrm{NH}_{4}\right)_{2} \mathrm{SO}_{4}$ foi utilizada em proporções estequiométricas em relação à massa de $\mathrm{H}_{2}{ }^{34} \mathrm{SO}_{4}$. A solução resultante foi colocada no balão de reação do destilador onde ocorreu a reação (1) com a adição de $20 \mathrm{~mL}$ solução $15 \mathrm{~mol} \mathrm{~L}^{-1}$ de $\mathrm{NaOH}$ (excesso). $\mathrm{A}^{15} \mathrm{NH}_{3(\text { aq) }}$ produzida foi arrastada pelo vapor de água e recebida em solução de $\mathrm{H}_{2}^{34} \mathrm{SO}_{4}$, contida em um recipiente acrílico, sob agitação, sendo o volume de ácido calculado pela estequiometria das reações (1) e (2). Por medida de segurança utilizou-se um medidor de $\mathrm{pH}$, para monitorar a acidez da solução resultante (reação 2), evitando-se, deste modo, possíveis perdas de ${ }^{15} \mathrm{NH}_{3}$. O final do processo foi identificado pela manutenção do $\mathrm{pH}$ na solução contendo

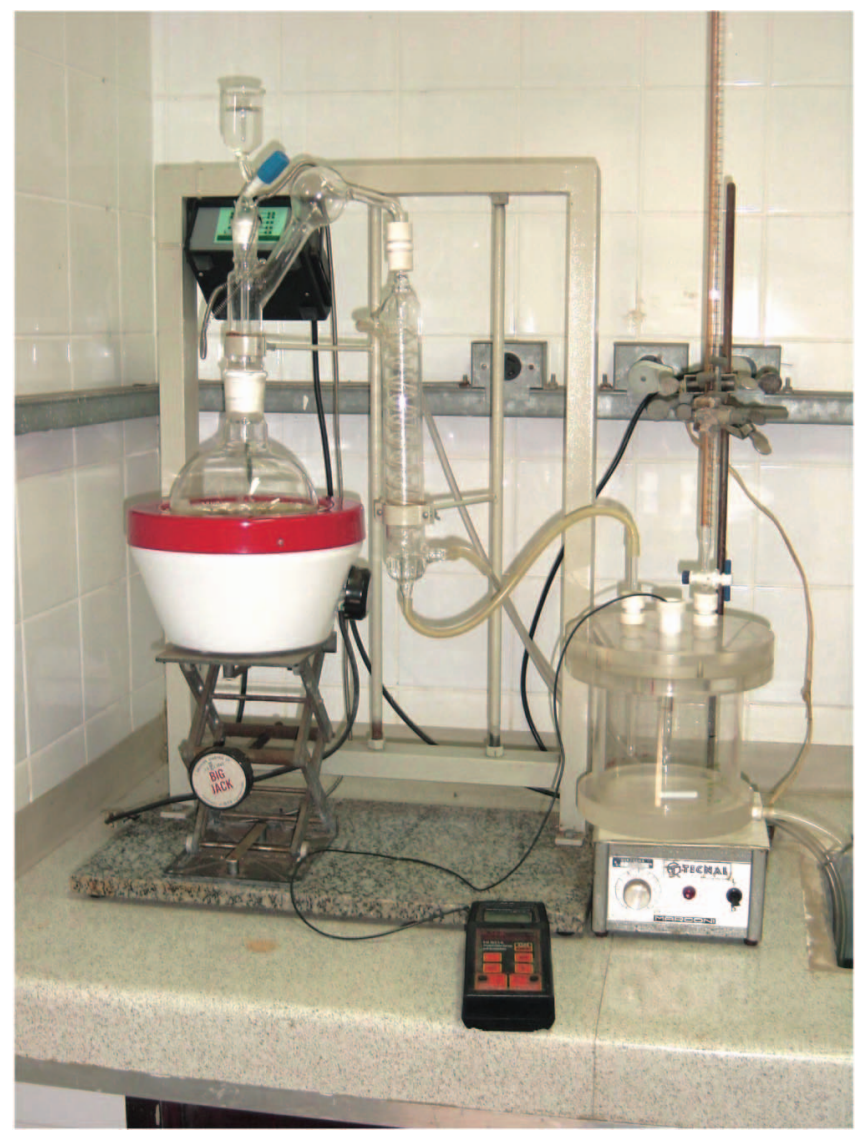

Figura 3. Sistema de produção de $\left({ }^{15} \mathrm{NH}_{4}\right)^{34} \mathrm{SO}_{4}$ a partir do $\left({ }^{15} \mathrm{NH}_{4}\right)_{2} \mathrm{SO}_{4}$ e $\mathrm{H}_{2}^{34} \mathrm{SO}_{4}$

$\left({ }^{15} \mathrm{NH}_{4}\right)_{2}^{34} \mathrm{SO}_{4}$, indicando a neutralização total da amônia- ${ }^{15} \mathrm{~N}$. O ajuste final do $\mathrm{pH}$ na faixa de 5,5 a 6,0 foi realizado, quando necessário, pela adição de pequenas quantidades de ${ }^{15} \mathrm{NH}_{3 \text { aq }}$ ou $\mathrm{H}_{2}{ }^{34} \mathrm{SO}_{4}$ diluído. $\mathrm{Na}$ última etapa a solução contendo o composto duplamente marcado $\left(\left({ }^{15} \mathrm{NH}_{4}\right)_{2}^{34} \mathrm{SO}_{4}\right)$ foi transferida para o sistema de secagem à temperatura de $50{ }^{\circ} \mathrm{C}$, sendo o sal amoniacal cristalizado.

\section{RESULTADOS E DISCUSSÃO}

\section{Produção de $\left({ }^{15} \mathrm{NH}_{4}\right){ }_{2}^{34} \mathrm{SO}_{4}$ por troca iônica}

A saturação dos sítios ativos da resina à forma $\mathrm{NH}_{4}^{+}\left(\mathrm{R}-\mathrm{H}^{+} \rightarrow \mathrm{R}-\mathrm{NH}_{4}^{+}\right)$ foi obtida com $2700 \mathrm{~mL}$ de solução $0,5 \mathrm{~mol} \mathrm{~L}^{-1}$ de $\left({ }^{15} \mathrm{NH}_{4}\right)_{2} \mathrm{SO}_{4}$ com fluxo de aproximadamente $2 \mathrm{~mL} \mathrm{~cm}^{-2} \mathrm{~min}^{-1}$. Este volume de $\left({ }^{15} \mathrm{NH}_{4}\right)_{2} \mathrm{SO}_{4}$ foi determinado pela quantificação do $\mathrm{NH}_{4}{ }^{+}$nas frações coletadas no volume eluído (alíquotas de $100 \mathrm{~mL}$ ). Neste ponto $(2700 \mathrm{~mL})$ a relação entre a concentração de $\mathrm{NH}_{4}^{+}$no afluente (solução $0,5 \mathrm{~mol} \mathrm{~L}^{-1}$ de $\mathrm{NH}_{4}^{+}$) e do volume eluído foi aproximadamente igual a 1, indicando a saturação ou carga dos sítios ativos da resina à forma $\mathrm{NH}_{4}^{+}\left(\mathrm{R}_{-} \mathrm{NH}_{4}^{+}\right)$. A solução eluída foi totalmente coletada para posterior recuperação do $\left({ }^{15} \mathrm{NH}_{4}\right)_{2} \mathrm{SO}_{4}$, que correspondeu a aproximadamente $100 \mathrm{~g}$.

Numa etapa seguinte, como descrito anteriormente, foi realizada a lavagem para eliminação completa do $\left({ }^{15} \mathrm{NH}_{4}\right)_{2} \mathrm{SO}_{4}$ remanescente no volume intersticial da resina, sendo necessário $300 \mathrm{~mL}$ de água desionizada, no qual pôde-se recuperar 14,3 $\pm 0,5 \mathrm{~g}$ de $\left({ }^{15} \mathrm{NH}_{4}\right)_{2} \mathrm{SO}_{4}$.

$\mathrm{Na}$ etapa seguinte utilizou-se de solução de $\mathrm{Na}_{2}{ }^{34} \mathrm{SO}_{4}(30,50 \mathrm{e}$ $70 \mathrm{~g} \mathrm{~L}^{-1}$ ) como eluente dos íons $\mathrm{NH}_{4}^{+}$. A troca entre os íons $\mathrm{Na}^{+}(\mathrm{s})$ e $\mathrm{NH}_{4}^{+}(\mathrm{r})$ possibilitou a formação do $\left({ }^{15} \mathrm{NH}_{4}\right)_{2}{ }^{34} \mathrm{SO}_{4}$ no eluído.

A massa de $\left({ }^{15} \mathrm{NH}_{4}\right)_{2}{ }^{34} \mathrm{SO}_{4}$ no volume útil (levando-se em consideração a concentração de $\mathrm{Na}^{+}$) a partir da eluição do $\mathrm{NH}_{4}^{+}$dos sítios 
Tabela 1. Balanço de massa na produção de $\left({ }^{15} \mathrm{NH}_{4}\right)_{2}^{34} \mathrm{SO}_{4}$ por troca catiônica

\begin{tabular}{|c|c|c|c|c|c|}
\hline $\begin{array}{c}\text { Eluente } \\
\mathrm{Na}_{2}{ }^{34} \mathrm{SO}_{4} \\
\left(\mathrm{~g} \mathrm{~L} \mathrm{~L}^{-1}\right)\end{array}$ & $\begin{array}{l}\text { Volume } \\
\text { eluído }^{(1)} \\
\text { (mL) }\end{array}$ & $\begin{array}{c}\text { Massa de } \\
\left({ }^{15} \mathrm{NH}_{4}\right)_{2}^{34} \mathrm{SO}_{4} \\
\text { produzida }^{(2)}(\mathrm{g})\end{array}$ & $\begin{array}{c}\text { Massa de } \mathrm{Na}^{+} \\
\text {no volume } \\
(\mathrm{g})\end{array}$ & $\begin{array}{c}\text { Massa de } \\
\left({ }^{15} \mathrm{NH}_{4}\right)_{2}{ }^{34} \mathrm{SO}_{4} \\
\text { residual }^{(3)}(\mathrm{g})\end{array}$ & $\begin{array}{c}\left(\mathrm{NH}_{4}\right)_{2} \mathrm{SO}_{4} \\
\text { residual } \\
(\%)\end{array}$ \\
\hline 30 & 2.300 & $55 \pm 1$ & $1,6 \pm 0,3$ & $1,3 \pm 0,4$ & $2,3 \pm 0,7$ \\
\hline 50 & 1.400 & $54,1 \pm 0,6$ & $1,3 \pm 0,2$ & $2,9 \pm 0,6$ & $5 \pm 1$ \\
\hline 70 & 1.100 & $55,2 \pm 0,3$ & $1,8 \pm 0,1$ & $2,8 \pm 0,1$ & $4,9 \pm 0,1$ \\
\hline
\end{tabular}

${ }^{(1)}$ volume eluído com concentração de Na inferior a $1 \%(\mathrm{~m} / \mathrm{v})$; ${ }^{(2)}$ massa de $\left({ }^{15} \mathrm{NH}_{4}\right){ }_{2}^{34} \mathrm{SO}_{4}$ produzida no volume útil considerando o balanço sulfato de amônio e sódio; ${ }^{(3)}$ massa de $\left({ }^{15} \mathrm{NH}_{4}\right){ }_{2}^{34} \mathrm{SO}_{4}$ contendo $\mathrm{Na}^{+}$com concentração superior a $1 \%(\mathrm{~m} / \mathrm{v})$. Os resultados representam a média e desvio padrão da média de 3 repetições $(n=3)$.

ativos da resina, com solução de $\mathrm{Na}_{2}{ }^{34} \mathrm{SO}_{4}$, nas concentrações (30, 50 e $70 \mathrm{~g} \mathrm{~L}^{-1}$ ), pode ser visualizada na Tabela 1. São apresentados, também, o volume eluído recuperado, a massa de sódio no volume útil considerado, a massa de $\left({ }^{15} \mathrm{NH}_{4}\right)_{2}{ }^{34} \mathrm{SO}_{4}$ residual (não considerado no volume útil) e a porcentagem do resíduo em relação a massa total de $\left({ }^{15} \mathrm{NH}_{4}\right)_{2}{ }^{34} \mathrm{SO}_{4}$.

As curvas de eluição do ${ }^{15} \mathrm{NH}_{4}^{+}$, para as três concentrações de eluente, estão representadas na Figura 4, onde se verifica a variação da concentração de $\mathrm{Na}^{+}$em função do volume eluído.

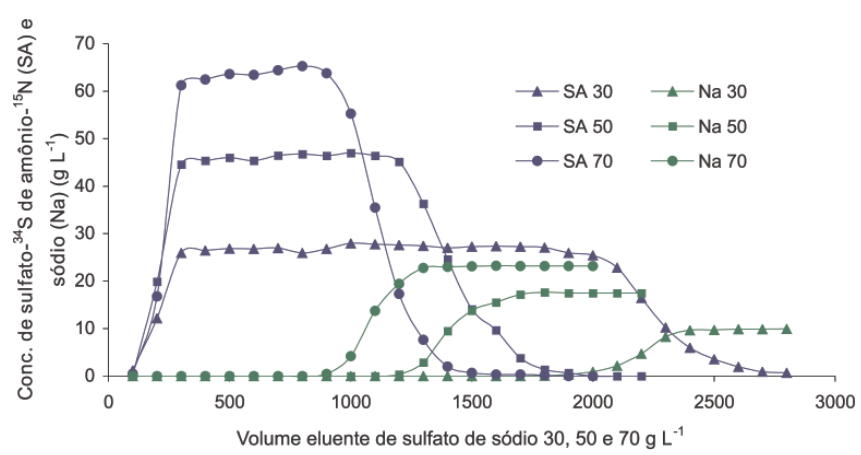

Figura 4. Curva de eluição do $\mathrm{NH}_{4}^{+}$e produção de $\left({ }^{15} \mathrm{NH}_{4}\right){ }_{2}^{34} \mathrm{SO}_{4}$

A resina catiônica apresenta maior seletividade para o $\mathrm{Na}^{+}$em relação ao $\mathrm{NH}_{4}^{+31,32}$, favorecendo o processo de troca envolvendo o deslocamento do íon $\mathrm{NH}_{4}^{+}$pelo $\mathrm{Na}^{+}$(íon trocador). Concluída a eluição, a concentração do íon $\mathrm{NH}_{4}^{+}$foi inversamente proporcional à do íon $\mathrm{Na}^{+}$. A Figura 4 apresenta a eluição dos íons ${ }^{15} \mathrm{NH}_{4}^{+}$que é finalizada após a utilização de 2800, 2000 e $1600 \mathrm{~mL}$ da solução eluente de $\mathrm{Na}_{2}{ }^{34} \mathrm{SO}_{4}$, para as concentrações de 30,50 e $70 \mathrm{~g} \mathrm{~L}^{-1}$, respectivamente.

$\mathrm{O}$ início da produção do composto $\left({ }^{15} \mathrm{NH}_{4}\right)_{2}^{34} \mathrm{SO}_{4}$, para todas as concentrações avaliadas do eluente, foi constatada a partir do volume de 200 mL e a concentração máxima do sal no eluído foi atingida a partir de $300 \mathrm{~mL}$. Após este volume notou-se que a produção se manteve praticamente constante, até atingir o ponto de inflexão de cada curva, quando ocorreu o ponto de ruptura do íon trocador $\mathrm{Na}^{+}$.

A curva de ruptura ${ }^{33}$ foi caracterizada pelo início da detecção do trocador $\mathrm{Na}^{+}$no eluído e indicou o momento de início da saturação dos sítios ativos da resina pelos íons $\mathrm{Na}^{+}$. Esta etapa ocorreu aos 1900, 1200 e $900 \mathrm{~mL}$ do eluído, respectivamente, para as concentrações de 30, 50 e $70 \mathrm{~g} \mathrm{~L}^{-1}$. A partir desse ponto o eluído passou a conter o $\mathrm{Na}^{+}\left(\mathrm{Na}_{2}{ }^{34} \mathrm{SO}_{4}\right)$, além do $\left({ }^{15} \mathrm{NH}_{4}\right)_{2}{ }^{34} \mathrm{SO}_{4}$. A determinação do ponto de ruptura é um indicativo da pureza do sal $\left({ }^{15} \mathrm{NH}_{4}\right)_{2}{ }^{34} \mathrm{SO}_{4}$ produzido. Desta forma, tornou-se importante saber qual o nível de pureza necessário para o $\left({ }^{15} \mathrm{NH}_{4}\right)_{2}^{34} \mathrm{SO}_{4}$ ou até que nível de contaminação pode ser tolerado para o tipo de aplicação a que se destina. Nos resultados da Tabela 1 foram considerados os volumes eluentes que apresentaram quantidade de $\mathrm{Na}^{+}$inferiores a $1 \%(\mathrm{~m} / \mathrm{v})$. Assim, para o teste com o eluente $\mathrm{Na}_{2}{ }^{34} \mathrm{SO}_{4} 30 \mathrm{~g} \mathrm{~L}^{-1}$, foram considerados os primeiros $2300 \mathrm{~mL}$, como volume útil na produção do $\left({ }^{15} \mathrm{NH}_{4}\right){ }_{2}^{34} \mathrm{SO}_{4}$. A partir dos $2300 \mathrm{~mL}$ (fração 2300 a $2400 \mathrm{~mL}$ ) o teor de $\left({ }^{15} \mathrm{NH}_{4}\right)_{2}^{34} \mathrm{SO}_{4}$ foi muito baixo e a concentração de $\mathrm{Na}^{+}$no eluído $\left(9,64 \mathrm{~g} \mathrm{~L}^{-1} \mathrm{de} \mathrm{Na}^{+}\right.$, equivalente a $29,75 \mathrm{~g} \mathrm{~L}^{-1} \mathrm{Na}_{2}{ }^{34} \mathrm{SO}_{4}$ ) foi praticamente a mesma da solução eluente ( $\left.30 \mathrm{~g} \mathrm{~L}^{-1}\right)$. O volume de eluente utilizado $(2300 \mathrm{~mL})$, para $450 \mathrm{~mL}$ de resina, representou um valor de 5,1 VL (volume leito), que pode se considerado uma boa relação para troca iônica.

Em relação à solução eluente $50 \mathrm{~g} \mathrm{~L}^{-1} \mathrm{de} \mathrm{Na}_{2}{ }^{34} \mathrm{SO}_{4}$, pode-se considerar que o volume de $1400 \mathrm{~mL}(3,1 \mathrm{VL})$ seria o ideal objetivando a produção de $\left({ }^{15} \mathrm{NH}_{4}\right){ }_{2}^{34} \mathrm{SO}_{4}$ com baixa concentração de sódio (Figura 4). A partir desse ponto, a concentração de sódio no eluído atingiu valores médios de $9,50 \mathrm{~g} \mathrm{~L}^{-1} \mathrm{de} \mathrm{Na}^{+}$, muito próximo ao obtido nos testes com solução $30 \mathrm{~g} \mathrm{~L}^{-1} \mathrm{de} \mathrm{Na}_{2}^{34} \mathrm{SO}_{4}$. O volume utilizado para eluir praticamente todo ${ }^{15} \mathrm{NH}_{4}^{+}$contido em $450 \mathrm{~mL}$ de resina foi adequado, representando 3,1 de VL.

Para os testes com solução eluente $70 \mathrm{~g} \mathrm{~L}^{-1} \mathrm{de} \mathrm{Na}_{2}{ }^{34} \mathrm{SO}_{4}$, pôde-se verificar que aproximadamente $1100 \mathrm{~mL}(2,4 \mathrm{VL})$ foram utilizados na produção de $\left({ }^{15} \mathrm{NH}_{4}\right){ }_{2}^{34} \mathrm{SO}_{4}$, (Figura 4). Nessas condições verificou-se que a concentração média de $\mathrm{Na}^{+}$foi de $13,7 \mathrm{~g} \mathrm{~L}^{-1}(1,37 \mathrm{~g}$ / $100 \mathrm{~mL}$ ). A eluição com $\mathrm{Na}_{2}{ }^{34} \mathrm{SO}_{4} 70 \mathrm{~g} \mathrm{~L}^{-1}$ foi excepcionalmente boa, considerando-se que praticamente todo ${ }^{15} \mathrm{NH}_{4}^{+}$foi eluído com $1100 \mathrm{~mL}$ em $450 \mathrm{~mL}$ de resina, que representou um ótimo valor de volume leito $(2,4 \mathrm{VL})$. Os $100 \mathrm{~mL}$ finais, referentes ao intervalo de 1000 a $1100 \mathrm{~mL}$ com concentração de $\mathrm{Na}^{+}$de aproximadamente $0,9 \%$ $(\mathrm{m} / \mathrm{v})$, superior à encontrada nos testes anteriores $\left(30\right.$ e $\left.50 \mathrm{~g} \mathrm{~L}^{-1}\right)$, foi considerada em razão da expressiva massa de $\left({ }^{15} \mathrm{NH}_{4}\right)_{2}{ }^{34} \mathrm{SO}_{4}$ contida neste volume (aproximadamente 4,55 g).

Nos testes com as três diferentes concentrações da solução eluente, pôde-se verificar que a massa de $\left({ }^{15} \mathrm{NH}_{4}\right)_{2}{ }^{34} \mathrm{SO}_{4}$ residual recuperada, não considerada no processo de produção, foi de 1,$3 ; 2,9$ e $2,8 \mathrm{~g}$, respectivamente, para as concentrações de 30,50 e $70 \mathrm{~g} \mathrm{~L}^{-1}$ de $\mathrm{Na}_{2}{ }^{34} \mathrm{SO}_{4}$ (Tabela 1).

A produção média de $\left({ }^{15} \mathrm{NH}_{4}\right){ }_{2}^{34} \mathrm{SO}_{4}$ foi de $55 \pm 1 \mathrm{~g} ; 54,1 \pm 0,6 \mathrm{~g}$ e 55,2 $\pm 0,3 \mathrm{~g}$ nos testes utilizando $\mathrm{Na}^{34} \mathrm{SO}_{4}$ com concentração de 30,50 e $70 \mathrm{~g} \mathrm{~L}^{-1}$, respectivamente (Tabela 1). Esses valores mostram que a produção de $\left({ }^{15} \mathrm{NH}_{4}\right)_{2}^{34} \mathrm{SO}_{4}$, por troca iônica, não se mostrou dependente da concentração do eluente $\left(\mathrm{Na}_{2}{ }^{34} \mathrm{SO}_{4}\right)$. No entanto, o volume útil (concentração de $\mathrm{Na}^{+}$inferior a $1 \% \mathrm{~m} / \mathrm{v}$ ) de solução de $\mathrm{Na}_{2}{ }^{34} \mathrm{SO}_{4}$, necessário para eluir os íons $\mathrm{NH}_{4}{ }^{+}$dos sítios ativos da resina catiônica, foi em média de 2300, 1400 e $1100 \mathrm{~mL}$, respectivamente, para as concentrações de 30,50 e $70 \mathrm{~g} \mathrm{~L}^{-1} \mathrm{de} \mathrm{Na}_{2}{ }^{34} \mathrm{SO}_{4}$. Desta forma, considerando o volume útil, pôde-se determinar que a massa de $\mathrm{Na}_{2}{ }^{34} \mathrm{SO}_{4}$ necessária para a eluição dos íons $\mathrm{NH}_{4}{ }^{+}$da resina catiônica foi de 69,70 e $77 \mathrm{~g}$, respectivamente, para as soluções de 30,50 e $70 \mathrm{~g} \mathrm{~L}^{-1}$ de $\mathrm{Na}_{2}{ }^{34} \mathrm{SO}_{4}$.

Deve-se destacar que a completa eluição dos íons $\mathrm{NH}_{4}^{+}$retidos nos sítios ativos da resina, demandou 2700, 2000 e $1600 \mathrm{~mL}$ para as concentrações de 30,50 e $70 \mathrm{~g} \mathrm{~L}^{-1}$ de $\mathrm{Na}_{2}{ }^{34} \mathrm{SO}_{4}$, respectivamente. A massa de $\left({ }^{15} \mathrm{NH}_{4}\right){ }_{2}^{34} \mathrm{SO}_{4}$ residual, contida no volume final da eluição 
(400, 600 e $500 \mathrm{~mL}$ com relação às concentrações de 30,50 e $70 \mathrm{~g} \mathrm{~L}^{-1}$ de $\mathrm{Na}_{2}{ }^{34} \mathrm{SO}_{4}$, respectivamente), foi quantitativamente recuperada em sistema de destilação ( Figura 3).

Considerando um fluxo de 5 a $6 \mathrm{~mL} \mathrm{~min}^{-1}$, pôde-se determinar que o tempo de duração do processo completo para os testes com concentração de $30 \mathrm{~g} \mathrm{~L}^{-1}$ foi de aproximadamente $8 \mathrm{~h}$, gastando-se $4 \mathrm{~h}$ a mais que no processo em que se utilizou a concentração de $50 \mathrm{~g} \mathrm{~L}^{-1}(4 \mathrm{~h})$. Com os dados da Tabela 1, pode-se observar que a produção de $\left({ }^{15} \mathrm{NH}_{4}\right)_{2}^{34} \mathrm{SO}_{4}$ é praticamente a mesma, para as três concentrações de eluente avaliadas, e o consumo de $\mathrm{Na}_{2}{ }^{34} \mathrm{SO}_{4}$ foi inferior quando se utilizou o eluente com concentração de 30 e $50 \mathrm{~g} \mathrm{~L}^{-1}$ (69 e 70 g). Desta forma, levando-se em consideração o tempo de produção, a solução eluente com concentração de $50 \mathrm{~g} \mathrm{~L}^{-1}$ de $\mathrm{Na}_{2}{ }^{34} \mathrm{SO}_{4}$ foi a mais indicada.

A última etapa do processo de produção de $\left({ }^{15} \mathrm{NH}_{4}\right)_{2}{ }^{34} \mathrm{SO}_{4}$ consistiu na lavagem do $\mathrm{Na}_{2}{ }^{34} \mathrm{SO}_{4}$, do volume intersticial da coluna de resina, com água desionizada, recuperando-se o composto, principalmente, quando da utilização de ${ }^{34} \mathrm{~S}$. Nessa etapa foram necessários de 400 a $500 \mathrm{~mL}$ de $\mathrm{H}_{2} \mathrm{O}$ desionizada, sendo possível a recuperação de $2,4 \pm$ 0,$1 ; 3,5 \pm 0,1$ e 4,8 $\pm 0,1 \mathrm{~g}$ de $\mathrm{Na}_{2}{ }^{34} \mathrm{SO}_{4}$ quando se utilizou a solução eluente com concentrações de 30,50 e $70 \mathrm{~g} \mathrm{~L}^{-1}$, respectivamente.

Em teste empregando os reagentes $\mathrm{Na}_{2}^{34} \mathrm{SO}_{4}$ e $\left({ }^{15} \mathrm{NH}_{4}\right)_{2} \mathrm{SO}_{4}$, previamente enriquecidos nos isótopos ${ }^{34} \mathrm{~S}$ e ${ }^{15} \mathrm{~N}(8,40$ e $15,2 \%$ em átomos de ${ }^{34} \mathrm{~S}$ e ${ }^{15} \mathrm{~N}$, respectivamente), foi possível a obtenção de aproximadamente $54,5 \mathrm{~g}$ de $\left({ }^{15} \mathrm{NH}_{4}\right){ }_{2}^{34} \mathrm{SO}_{4}$ com marcação de 8,39 e $15,2 \%$ em átomos de ${ }^{34} \mathrm{~S}$ e ${ }^{15} \mathrm{~N}$. Estes resultados mostraram que não ocorre fracionamento isotópico durante o processo de troca iônica na produção de sulfato de amônio duplamente marcado, pois o produto final apresentou a mesma abundância isotópica $\left({ }^{34} \mathrm{~S}\right.$ e $\left.{ }^{15} \mathrm{~N}\right) \operatorname{dos}$ reagentes iniciais.

\section{Produção de $\mathrm{H}_{2}^{34} \mathrm{SO}_{4}$ por troca iônica e produção de $\left({ }^{15} \mathrm{NH}_{4}\right)_{2}{ }^{34} \mathrm{SO}_{4}$ na reação entre ${ }^{15} \mathrm{NH}_{3(\mathrm{aq})}$ e $\mathrm{H}_{2}{ }^{34} \mathrm{SO}_{4}$}

Os sítios ativos da resina catiônica Dowex 50w X8 foram saturados com $\mathrm{H}^{+}$, com aproximadamente $3000 \mathrm{~mL}$ de solução 0,5 mol L ${ }^{-1}$ de $\mathrm{H}_{2} \mathrm{SO}_{4}$. $\mathrm{O}$ excesso de $\mathrm{H}_{2} \mathrm{SO}_{4}$ do volume intersticial foi lavado com $3000 \mathrm{~mL}$ de água desionizada. Após a descompactação da resina (retrolavagem com $\mathrm{H}_{2} \mathrm{O}$ desionizada), pôde-se determinar que o volume da resina na forma $\mathrm{H}^{+}\left(\mathrm{R}-\mathrm{H}^{+}\right)$e equilibrada em água foi de aproximadamente $450 \mathrm{~mL}$.

$\mathrm{Na}$ etapa seguinte os íons $\mathrm{H}^{+}$, adsorvidos nos sítios ativos da resina, foram eluídos com soluções 30,50 e $70 \mathrm{~g} \mathrm{~L}^{-1}$ de $\mathrm{Na}_{2}{ }^{34} \mathrm{SO}_{4}$ (eluente), produzindo $\mathrm{H}_{2}{ }^{34} \mathrm{SO}_{4}$ no eluído. Os testes foram realizados em triplicata para cada uma das concentrações do eluente. As três concentrações de eluente, o volume e a massa de eluente utilizado, a massa de $\mathrm{H}_{2}{ }^{34} \mathrm{SO}_{4}$ obtida, a massa de $\left({ }^{15} \mathrm{NH}_{4}\right)_{2}{ }^{34} \mathrm{SO}_{4}$ produzida e a \% de $\mathrm{H}_{2}{ }^{34} \mathrm{SO}_{4}$ e $\mathrm{Na}_{2}{ }^{34} \mathrm{SO}_{4}$ obtidas a partir da massa de eluente empregado (volume útil) constam da Tabela 2.

O volume e a massa do eluente corresponderam ao montante com baixa concentração de $\mathrm{Na}(<1 \% \mathrm{~m} / \mathrm{v})$ no eluído e é denominado de volume útil. Para a concentração de $30 \mathrm{~g} \mathrm{~L}^{-1}$ de $\mathrm{Na}_{2}{ }^{34} \mathrm{SO}_{4}$ este volume foi em média de $2275 \mathrm{~mL}$, de um total eluído de $2500 \mathrm{~mL}$. Nos 225 $\mathrm{mL}$ finais tem-se aproximadamente $0,15 \mathrm{~g} \mathrm{de} \mathrm{H}_{2}{ }^{34} \mathrm{SO}_{4}$, correspondendo a apenas $0,3 \%$ do total eluído. Entretanto, nesse volume final a concentração de $\mathrm{Na}^{+}$foi da ordem de $11,0 \mathrm{~g} \mathrm{~L}^{-1}$, enquanto que no volume útil ficou em aproximadamente $0,5 \mathrm{~g} \mathrm{~L}^{-1} \mathrm{de} \mathrm{Na}(0,05 \%)$. Nos experimentos com concentração do eluente de 50 e $70 \mathrm{~g} \mathrm{~L}^{-1} \mathrm{de} \mathrm{Na}_{2}{ }^{34} \mathrm{SO}_{4}$, o volume útil foi de 1475 e $1100 \mathrm{~mL}$, respectivamente. A produção de $\mathrm{H}_{2}^{34} \mathrm{SO}_{4}$, para as três concentrações do eluente foi muito semelhante, sendo da ordem de 42 a $43 \mathrm{~g}$ do ácido (Tabela 2).

Nos ensaios empregando solução eluente $50 \mathrm{~g} \mathrm{~L}^{-1} \mathrm{de} \mathrm{Na}_{2}{ }^{34} \mathrm{SO}_{4}$, verificou-se uma excelente eluição (VL de 3,3) com produção média de 43,04 \pm 0,01 g de $\mathrm{H}_{2}{ }^{34} \mathrm{SO}_{4}$ (solução $0,3 \mathrm{~mol} \mathrm{~L}^{-1} \mathrm{de}_{2}{ }^{34} \mathrm{SO}_{4}$ ) e com concentração, no volume útil, de $0,4 \mathrm{~g} \mathrm{~L}^{-1}$ de $\mathrm{Na}$. Nessas condições observou-se um maior percentual $(86,00 \pm 0,03 \%)$ de conversão do sal $\mathrm{Na}_{2}{ }^{34} \mathrm{SO}_{4}$ (eluente) para o ácido $\left(\mathrm{H}_{2}^{34} \mathrm{SO}_{4}\right)$ (Tabela 2). Considerando o volume útil de $1475 \mathrm{~mL}$ para o eluente $(73,8 \mathrm{~g})$ e fluxo de $6 \mathrm{ml} \mathrm{min}{ }^{-1}\left(1,7 \mathrm{~mL} \mathrm{~cm}^{-2} \mathrm{~min}^{-1}\right)$, o tempo consumido nesta etapa foi da ordem de $4 \mathrm{~h}$. Pôde-se verificar, ainda, que o processo foi muito eficiente não apresentando perdas, principalmente quando se utilizou material enriquecido nos isótopos ${ }^{15} \mathrm{~N}$ e ${ }^{34} \mathrm{~S}$.

Com o sistema proposto e utilizando-se de $74 \mathrm{~g} \mathrm{de} \mathrm{Na}_{2}{ }^{34} \mathrm{SO}_{4}$ enriquecido com $9,8 \%$ em átomos de ${ }^{34} \mathrm{~S}$ foi possível obter $43,1 \mathrm{~g} \mathrm{de}$ $\mathrm{H}_{2}^{34} \mathrm{SO}_{4}(1475 \mathrm{~mL})$ com a mesma abundância isotópica do eluente $\left(9,8 \%\right.$ átomos de $\left.{ }^{34} \mathrm{~S}\right)$.

Partindo-se de 58,1 g de $\left({ }^{15} \mathrm{NH}_{4}\right)_{2} \mathrm{SO}_{4}(0,44$ mols) enriquecido em $10 \%$ em átomos de ${ }^{15} \mathrm{~N}$ (como obtido no laboratório de Isótopos Estáveis do $\left.\mathrm{CENA}^{32,33}\right)$ e de $43,1 \mathrm{~g}$ de $\mathrm{H}_{2}{ }^{34} \mathrm{SO}_{4}(0,44$ mols) com enriquecimento isotópico de $9,8 \%$ átomos de ${ }^{34} \mathrm{~S}$, obtida no presente trabalho e, utilizando-se do sistema de destilação apresentado na Figura 3, foi possível a obtenção de sulfato de amônio duplamente marcado $\left(\left({ }^{15} \mathrm{NH}_{4}\right)_{2}{ }^{34} \mathrm{SO}_{4}\right)$. As reações estequiométricas para a produção do composto $\left({ }^{15} \mathrm{NH}_{4}\right)_{2}^{34} \mathrm{SO}_{4}$ estão representadas nas Equações (1) e (2).

$\mathrm{O}$ sal de $\left({ }^{15} \mathrm{NH}_{4}\right)_{2} \mathrm{SO}_{4}$ foi solubilizado em $300 \mathrm{~mL}$ de água $(58,1 \mathrm{~g}$ do composto enriquecido a $10 \%$ em átomos de ${ }^{15} \mathrm{~N}$ ) e a solução foi colocada em balão de reação do destilador (Figura 3), onde ocorreu a reação entre o $\left({ }^{15} \mathrm{NH}_{4}\right)_{2} \mathrm{SO}_{4}$ e $\mathrm{NaOH}$ (reação 1).

$\mathrm{O}$ procedimento foi realizado em aproximadamente 45 min e utilizou-se de 43,1 g de $\mathrm{H}_{2}{ }^{34} \mathrm{SO}_{4}$ contido em $1500 \mathrm{~mL}$ de solução $\left(0,3 \mathrm{~mol} \mathrm{~L}^{-1}\right)$ para neutralização da ${ }^{15} \mathrm{NH}_{3 \text { (aq) }}$. A solução final apresentou $\mathrm{pH}$ de 5,8 e volume de $1900 \mathrm{~mL}$. Após secagem do sal, em chapa aquecedora a $50{ }^{\circ} \mathrm{C}$ obteve-se $58,0 \mathrm{~g}$ de $\left({ }^{15} \mathrm{NH}_{4}\right)_{2}{ }^{34} \mathrm{SO}_{4}$ enriquecido a 10,0 e $9,8 \%$ em átomos de ${ }^{15} \mathrm{~N}$ e ${ }^{34} \mathrm{~S}$, respectivamente.

\section{CONCLUSÃO}

Para a produção de $\left({ }^{15} \mathrm{NH}_{4}\right)_{2}{ }^{34} \mathrm{SO}_{4}$ em sistema de cromatografia de troca catiônica, a utilização de solução de $\mathrm{Na}_{2}{ }^{34} \mathrm{SO}_{4}\left(50 \mathrm{~g} \mathrm{~L}^{-1}\right)$ foi adequada. Partindo-se de $70 \mathrm{~g}$ de $\mathrm{Na}_{2}{ }^{34} \mathrm{SO}_{4}$ e $64,2 \mathrm{~g}$ de $\left({ }^{15} \mathrm{NH}_{4}\right)_{2} \mathrm{SO}_{4}$ foi possível obter $54,1 \mathrm{~g}$ de $\left({ }^{15} \mathrm{NH}_{4}\right)_{2}^{34} \mathrm{SO}_{4}$. A metodologia empregada mostrou-se viável e o composto com dupla marcação poderá ser

Tabela 2. Produção de $\left({ }^{15} \mathrm{NH}_{4}\right)_{2}^{34} \mathrm{SO}_{4}$ a partir do $\mathrm{H}_{2}{ }^{34} \mathrm{SO}_{4}$ e $\left({ }^{15} \mathrm{NH}_{4}\right)_{2} \mathrm{SO}_{4}$

\begin{tabular}{|c|c|c|c|c|c|}
\hline \multirow{2}{*}{$\begin{array}{c}\text { Eluente } \\
\mathrm{Na}_{2}{ }^{34} \mathrm{SO}_{4} \\
\left(\mathrm{~g} \mathrm{~L}^{-1}\right)\end{array}$} & \multirow{2}{*}{$\begin{array}{l}\text { Volume/massa } \\
\text { eluente utilizado } \\
\qquad(\mathrm{ml} / \mathrm{g})\end{array}$} & \multirow{2}{*}{$\begin{array}{c}\text { Produção de } \\
\mathrm{H}_{2}{ }^{34} \mathrm{SO}_{4} \\
(\mathrm{~g})\end{array}$} & \multirow{2}{*}{$\begin{array}{c}\text { Produção de } \\
\left({ }^{15} \mathrm{NH}_{4}\right)_{2}{ }^{34} \mathrm{SO}_{4} \\
(\mathrm{~g})\end{array}$} & \multicolumn{2}{|c|}{$\mathrm{Na}_{2}^{34} \mathrm{SO}_{4}$ inicial } \\
\hline & & & & $\begin{array}{c}\mathrm{H}_{2}{ }^{34} \mathrm{SO}_{4}^{(1)} \\
(\%)\end{array}$ & $\begin{array}{c}\mathrm{Na}_{2}{ }^{34} \mathrm{SO}_{4}^{(2)} \\
(\%)\end{array}$ \\
\hline 30 & $2275 / 68,3$ & $41,9 \pm 0,9$ & $57,0 \pm 1,0$ & $81 \pm 2$ & $19 \pm 2$ \\
\hline 50 & $1475 / 73,8$ & $43,04 \pm 0,01$ & $58,03 \pm 0,02$ & $86,00 \pm 0,03$ & $14,00 \pm 0,03$ \\
\hline 70 & $1100 / 77,0$ & $42,6 \pm 0,6$ & $57,4 \pm 0,8$ & $79 \pm 2$ & $21 \pm 2$ \\
\hline
\end{tabular}

(1) \% do $\mathrm{Na}_{2}{ }^{34} \mathrm{SO}_{4}$ inicial $\left(\mathrm{S}_{-}{ }^{34} \mathrm{SO}_{4}\right)$ obtido como $\mathrm{H}_{2}{ }^{34} \mathrm{SO}_{4} ;{ }^{(2)} \%$ de $\mathrm{Na}_{2}{ }^{34} \mathrm{SO}_{4}$ inicial $\left(\mathrm{S}^{34} \mathrm{SO}_{4}\right)$ recuperado como $\mathrm{Na}_{2}{ }^{34} \mathrm{SO}_{4}$ na água de lavagem. Os resultados representam a média e desvio padrão da média de 3 repetições $(n=3)$. 
obtido, com alto grau de pureza e com níveis de enriquecimento em ${ }^{15} \mathrm{Ne} \mathrm{e}^{34} \mathrm{~S}$ dependentes das concentrações desses isótopos nos compostos $\left({ }^{15} \mathrm{NH}_{4}\right)_{2} \mathrm{SO}_{4}$ e $\mathrm{Na}_{2}{ }^{34} \mathrm{SO}_{4}$.

A obtenção do $\left({ }^{15} \mathrm{NH}_{4}\right)_{2}{ }^{34} \mathrm{SO}_{4}$ a partir da reação de neutralização entre o $\mathrm{H}_{2}^{34} \mathrm{SO}_{4}$ e ${ }^{15} \mathrm{NH}_{3 \text { aq }}$ foi também eficiente. Neste processo foi possível produzir 58,0 g do composto duplamente marcado $\left({ }^{15} \mathrm{~N}\right.$ e ${ }^{34} \mathrm{~S}$ ) a partir de $43,1 \mathrm{~g} \mathrm{de} \mathrm{H}_{2}{ }^{34} \mathrm{SO}_{4}$ (obtido por troca iônica com 74,0 g de $\left.\mathrm{Na}_{2}{ }_{3}^{44} \mathrm{SO}_{4}\right)$ e de $58,1 \mathrm{~g}$ de $\left({ }^{15} \mathrm{NH}_{4}\right)_{2} \mathrm{SO}_{4}$.

Nos dois processos foi possível obter $0,78 \mathrm{~g}$ de $\left({ }^{15} \mathrm{NH}_{4}\right)_{2}{ }^{34} \mathrm{SO}_{4}$ para cada grama do eluente $\mathrm{Na}_{2}{ }^{34} \mathrm{SO}_{4}$.

O processo mostrou-se operacional e economicamente viável, sendo possível obter $\left({ }^{15} \mathrm{NH}_{4}\right)_{2}{ }^{34} \mathrm{SO}_{4}$ enriquecido a $10 \%$ em átomos de ${ }^{15} \mathrm{~N}$ e do ${ }^{34} \mathrm{~S}$ ao custo de US\$ 30.00 por grama.

\section{REFERÊNCIAS}

1. Leningher, A. L.; Fundamentos de bioquímica, Savier: São Paulo, 1980.

2. Zhao, F. J.; Verkampen, K. C. J.; Birdsey, M.; Blake-Kalff, M. M. A.; MaGrath, S. P.; J. Plant Nutr. 2001, 24, 1551.

3. Vitti, G. C.; Mutton, M. A.; Fornasiri Filho, D.; Perecin, D.; Rosolen, C. A.; Nociti, P. R. H.; Mendes, J. M.; Paranhos, S. B.; Piccin, C. R.; Malavolta, M. L.; Malavolta, E. Em Efeitos de Doses e Fontes de Enxofre em Culturas de Interesse Econômico; Malavolta, E., coord.; SN: Piracicaba, 1989.

4. Wood, A. W.; Soil Tittage Research 1991, 20, 69.

5. Vitti, A. C.; Tese de Doutorado, Universidade de São Paulo, Brasil, 2003.

6. Bull, L.T.; Cantarella, H.; Cultura do milho: Fatores que afetam a produtividade, Potafos: Piracicaba, 1993.

7. Rodrigues, M. B.; Kiehl, J. C.; Rev. Bras. Ci. Solo 1986, 10, 37.

8. Manechini, C.; Seminário Copersucar de Tecnologia Agronomica, Piracicaba, Brasil, 1997.

9. Trivelin, P. C. O.; Bendassolli, J. A.; Muraoka, T.; Carneiro Jr., F.; Sci. Agric. 2002, 59, 205.

10. Kiehl, J. C.; Rev. Bras. Ci. Solo 1989, 13, 75

11. Prammanee, P.; Bootun, A.; Lairungreong, C.; Thumthong, P.; Sruttaporn, C.; Thongyai, C.; Proceedings of 22 Internacional Society of Sugar Cane Tecnologist congress, Cartagena, Tecnicanã, 1996.

12. Denmead, O. T.; Freney, J. R.; Jackson, A. V.; Smith, J. W. B.; Saffigna, P. G.; Wood, A. W.; Chapman, L.S.; Proceedings of 12 a Australian Society of Sugar Cane Technologists, Brisbane, Austrália, 1990.
13. Trivelin, P. C. O.; Bendassolli, J. A.; Oliveira, M. W.; Muraoka, T.; STAB - Açúcar, Álcool e Subprodutos 1997, 16, 26.

14. Vitti, A. C.; Trivelin, P. C. O.; Gava, G. J. C.; Penatti, C. P.; Oliveira, M. W.; Anais do $8^{\circ}$ Congresso Nacional da Sociedade dos Técnicos Açucareiros e Alcooleiros do Brasil, Recife, Brasil, 2002.

15. Trivelin, P. C. O.; Bendassolli, J. A.; Muraoka, T.; Carneiro Jr., F.; Sci. Agr. 2002, 59, 205

16. Bissani, C. A.; Tedesco, M. J.; Resumos da 17 a Reunião Brasileira de fertilidade do solo, Londrina, Brasil, 1986.

17. Vitti, G. C.; Seminário fósforo, cálcio, magnésio, enxofre e micronutrientes, São Paulo: Brasil, 1986.

18. Teixeira, G. M.; Trivelin, P. C. O.; Bendassolli, J.A.; Muraoka, T.; Gava, G. J. C.; Anais do $6^{\circ}$ Encontro Nacional de Aplicações Nucleares, Rio de Janeiro, Brasil, 2002.

19. Lide, D. R.; Handbook of Chemistry and Physics, 78a ed., CRC Press: Boca Raton, 1997.

20. Charkson, D. T.; Smith, F. W.; J. Exp. Bot. 1983, 34, 1463

21. Larsson, C. M.; Larsson, M.; Purves, J. V.; Charkson, D. T.; Physiol. Plant 1991, 82, 345

22. Fitz-Gerald, M. A.; Ugalde, T. D.; Anderson, J. W.; J. Exp. Bot. 1999, 50, 499.

23. Patnaik, M. C.; Santhe, A.; J. Nucl. Agric. Biol. 1993, 22, 75.

24. Bansal, K. N.; Moziramani, D. P. J.; Nucl. Agric. Biol. 1993, 22, 42.

25. Bendassolli, J. A.; Trivelin, P. C. O.; Carneiro Jr., F.; J. Braz. Chem. Soc. 1997, 8, 13 .

26. Krouse, H. R.; Bernhard, M.; Schoenau, J. J.; Applications of Stable Isotope Techniques to Soil Sulfur Cycling in Mass Spectrometry of Soil, Marcel Dekker, Inc.: New York, 1996.

27. Hamilton, S. D.; Chalk, P. M.; Undovich, M. J.; Smith, C.; J. Appl. Radiat. Isot. 1991, 42, 1099.

28. Maximo, E.; Tese de Doutorado, Universidade de São Paulo, Brasil, 2002.

29. Maximo, E.; Bendassolli, J. A.; Trivelin, P. C. O.; Oliveira, C. R.; Rossete, A. L. R. M.; Abstracts of 4th International Conference on Isotope, Cape Town, África do Sul, 2002.

30. Maximo, E.; Bendassolli, J .A.; Trivelin, P. C. O.; Proceedings of $3^{\text {th }}$ International Conference on Isotope, Vancouver, Canada, 2000.

31. Collins, C. H.; Braga, G. L.; Banato, P. S.; Introdução a métodos cromatográficos, Unicamp: Campinas, 1997.

32. Harland, C. E.; Ion Exchange. Theory and Practice, The Royal Society of Chemistry: Cambridge, 1994.

33. Khym, J.X.; Analytical Ion-Exchange Procedures in Chemistry and Biology, Prentice-hall International Inc.; London, 1974. 